\title{
O PAPEL DA ENFERMEIRA NA ASSISTÊNCIA À GESTANTE SADIA
}

\author{
Nilcea Maria Neri Duarte * \\ Léa Cecilia Muxfeldt**
}

RBEn/04

DUARTE, N. M. N., MUXFELDT, L. C. - O papel da enfermeira na assistência à gestante sadia, Rev. Bras. Enf., R.J., 28 : :0-74, 1975.

\section{I - INTRODUÇÃO}

O presente trabalho tem por finalidade relatar a avaliação das atividades de enfermagem que foram planejadas e estão sendo executadas no Programa de Saúde Materna do Hospital de Clínicas de Porto Alegre (H.C.P.A.)

O programa de Saúde Materna foi basicamente implantado a nível de assistência ambulatorial. Participaram da organização uma Enfermeira de Saúde Pública, uma Enfermeira Obstétrica e os Obstetras que atuam na equipe.

Esta avaliação é referente ao período de fevereiro de 1973 a abril de 1975, e teve por finalidade:

1) identificar a qualidade do atendimento de enfermagem, tendo em vista a demanda, a cobertura, concentração e rendimento dos mesmos;

2) avaliar a repercussão de programas desta natureza junto às pacientes que se inscreveram para receber assistência no Ambulatório do Hospital.

\section{II - PROGRAMA DE SAÚDE MATERNA}

Para a organização do programa levou-se em consideração os seguintes conceitos:

- A assistência à gestante deve ser progressiva e integral. Para isto deve receber atenção suficiente e na intensidade desejada, de acordo com suas necessidades básicas e, portanto, por uma equipe multiprofissional.

- A prestação da assistência de enfermagem em atividades diretas e sistemáticas junto às pacientes, proporciona maior dinamização ao programa materno-infantil. Os danos redutíveis através de ações educativas, podem ser tratados a nível de atenção de enfermagem.

- Pelo seu preparo a enfermeira está apta para atuar com pacientes em

* Auxiliar de Ensiso da Escola de Enfermagem da U.F.R.S.

* Assistente de Ensino na Escola de Enfermagem da U.F.R.S. 
DUARTE, N. M. N., MUXFELDT, L. C. - O papel da enfermeira na assistência à gestante sadia, Rev. Bras. Enf., R.J., 28 : $70-74,1975$.

estado de saúde e de doença. Ela provê serviços diretos de enfermagem, mas também ensina às gestantes prepararem-se para o autocuidado.

\section{III - CARACTERÍSTICAS DA ASSIS- TÊNCIA À GESTANTE SADIA}

No Ambulatório de Pré-Natal do HCPA é proposto à gestante um atendimento mensal até o $7 .^{\circ}$ mês de gestação e do $7 .^{\circ}$ ao $9 .^{\circ}$, quinzenal, ou semanal, quando necessário.

A gestante é acompanhada através de:

- consulta à gestante de alto risco consulta médica;

- consulta à gestante sadia - consulta médica e consulta de enfermagem;

- entrevista à gestante de alto risco entrevista de enfermagem.

A entrevista à gestante de alto risco $\mathrm{e}$ a consulta de enfermagem à gestante sadia são realizadas por Enfermeira Obstétrica, com treinamento especial no serviço. A consulta de enfermagem é a atividade independente em que a gestante é atendida de forma sistemática e contínua, enquanto que a entrevista de enfermagem é a atividade interdependente em que a enfermeira supervisiona o seguimento da terapêutica médica, define e orienta os aspectos de educação relacionados aos problemas de saúde e à higiene pré-natal.

A inscrição da gestante no Serviço de Pré-Natal é realizada através da consulta médica, ocasião em que se procede a avaliação e encaminhamento das gestantes sadias, para a consulta de enfermagem, e das de alto risco para a entrevista de enfermagem. No atendimento à gestante compete à Enfermeira Obstétrica:

- fazer a inscrição da gestante;

- realizar a consulta de enfermagem à gestante sadia; realizar entrevista de enfermagem à gestante de alto risco;

- fazer controle de retornos;

- programar e promover trabalho de grupo com gestantes (CURSO PSICOPROFILÁTICO) .

\section{CONSULTA DE ENFERMAGEM}

CONSULTA - a CONSULTA DE ENFERMAGEM é a atenção de enfermagem prestada à gestante sadia, de modo sistemático e contínuo, tendo em vista o transcorrer de uma gestação, parto, puerpério e recém-nascido normais.

DURAÇÃO DA CONSULTA - está prevista para ser realizada em 30 minutos.

CONCENTRAÇÃO - a consulta de enfermagem é alternada com a consulta médica, tendo pois, $50 \%$ das consultas previstas para a gestante sadia.

AÇÕES COMPONENTES DA CONSUL$\mathrm{TA}$ - na realização da consulta, a enfermeira executa as seguintes ações:

$10^{\circ}$ - avaliação dos resultados dos exames laboratoriais realizados pela gestante;

$2 .^{\circ}$ - histćrico de enfermagem para identificação do perfil da gestante, visando orientá-la no atendimento às suas necessidades básicas e psico-sociais, baseada na investigação de:

- percepções e expectativas relacionadas à gestação;

- hábitos anteriores quanto ao sono, repouso, atividades físicas, recreação, alimentação, uso de fumo e álcool, eliminações e higiene pessoal;

- aspectos relacionados à sexualidade;

- aspectos relacionados a sinais e sintcmas na gestação;

- constelação familiar;

- atividade profissional;

- atividade na comunidade;

- condições sócio-econômicas;

- condições da habitação quanto à localização, tipo, dependências, ventila- 
DUARTE, N. M. N., MUXFFrDT, L. C. -- O papel da enfermeira na assistência à gestante sadia, Rev. Bras. Enf., R.J., 28 : 70-74, 1975.

ção, água, esgoto, instalação sanitária, lixo, luz, presença de animais e insetos.

3. ${ }^{\circ}$ - exame físico, que consta basicamente de:

- controle de pressão arterial;

- avaliação de estado nutricional da gestante, através do controle ponderal sistemático, da observação e de dados levantados no histórico de enfermagem, quanto aos hábitos alimentares;

- observação de alterações como edemas, varizes, erupções cutâneas, problemas de mamas...

4..$^{-}$- exame obstétrico, feito através de:

- medida da circunferência abdominal e altura uterina;

- execução de manobras de palpação;

- ausculta dos batimentos cardíacos fetais;

- toque vaginal, qrando indicado.

5..$^{\circ}$ - definição de problemas a nível de atenção de enfermagem e organização de um Plano Assistencial.

$6 .^{\circ}$ - orientação da gestante de acordo com os problemas identificados, considerando necessidades individuais, normas e rotinas do serviço.

7. ${ }^{\circ}$ - agendamento para imunização antitetânica e cursos desenvolvidos no serviçc.

$8^{\circ}$ - encaminhamento para outros locais (Laboratório, Serviço Social, Serviço Odontológico, Maternidade, Serviço de Puericultura, Clínica de Planejamento Familiar...).

9. - registro de consulta no prontuário.

No esquema de orientação prestada à gestante, foi dado ênfase a:

- Orientação e controle dietético. Tendo como base a identificação dos hábitos alimentares da gestante, de suas preferências, do poder aquisitivo da família, orienta-se quanto à importância da alimentação balanceada no período gestacional e é fornecido cardápio adequado quanto a tipos de alimentos, quantidade e freqüência. Para isso considera-se a utilização dos alimentos de fácil obtenção no meio. Desta forma realiza-se a promoção da saúde e prevenção de complicações materno-fetais;

- ensino e supervisão da gestante quanto aos cuidados básicos de higiene pré-natal, salientando-se higiene pessoal, do vestuário, atividade e repouso, divertimentos, atividades sexuais e higiene da habitação;

- orientação quanto à vacinação antitetânica, necessidade da mesma, número de doses, importância dos retornos. Agendamento para a aplicação do Anatox Tetânico;

- orientação quanto à identificação dos sinais e sintomas de trabalho de parto, conduta no parto e puerpério, com especial referência à revisão puerperal e planejamento familiar;

- orientação quanto à utilização dos recursos da Comunidade;

- informação quanto à assistência prestada ao recém-nascido, no Serviço de Puericultura do HCPA, motivando a paciente a realizar o registro precocemente;

- motivação e inscrição das pacientes para participação em atividades de grupo.

2. EDUCAÇÃO SANITÁRIA DE GRUPO - ao lado das atividades a nível individual, a enfermeira atua no desenvolvimento de cursos junto à gestante. Ł desenvolvido o CURSO PSICOPROFILÁTICO, que tem como objetivo oferecer condições para que as gestantes compreendam, aceitem e pratiquem conhecimentos adequados e atitudes quanto à gestação, parto, puerpério e recém-nascido. O desenvolvimento é feito através de 10 aulas teórico-práticas, em que é proporcionado à gestante informações sobre o desenvolvimento de uma gestação normal, quais os cuidados higiênicos no pré-natal, a influência do psiquismo sobre o físico, meios para conseguir re- 
DUARTE, N. M. N., MUXFELDT, L. C. - O papel da enfermeira na assistência à gestante sadia, Rev. Bras. Enf., R.J., 28 : $70-74,1975$.

laxamento, a conduta no trabalho de parto, parto e puerpério, bem como os primeiros cuidados ao recém-nascido, cuidados de higiene e alimentação. A informação teórica é complementada com demonstração prática e execução de exercícios adequados à gestação, tendo em visía reforçar e dar maior flexibilidade e elasticidade aos grupos musculares e articulações envolvidos no parto, capacitar a gestante ao auto-controle através dos exercícios de respiração e relaxamento. Atuam no desenvolvimento do curso a Enfermeira Obstétrica e a Enfermeira.

\section{AVALIAÇAO DAS ATIVIDADES DE ASSISTENCIA A GESTANTE SADIA}

Após 27 meses de funcionamento efetivo da atenção de enfermagem, a nível individual e de grupo, foi feita a avaliação, baseada nos relatórios da Unidade, levantamento de dados nos prontuários e preenchimento de questionários por gestante. Através da Consulta de Enfermagem foram identificados problemas, procurou-se levantar quais as que a enfermeira está capacitada a resolver, o percentual de retornos às consultas, as condições sócio-econômicas das gestantes inscritas e as imunizações realizadas.

\section{a) RESULTADOS OBTIDOS REFERENTES A CONSULTA DE ENFERMAGEM}

De fevereiro de 1973 a abril de 1975, foram inscritas, para receber atenção de enfermagem, 496 gestantes, com um total de 1.800 consultas, das quais $49,6 \%$ foram primeiras consultas e $58,4 \%$ seguimento.
Considerando o total de consultas oferecidas, que foi de 1.080 , registrou-se 80 feitas, o que corresponde a $7,40 \%$, ou seja, a $92,60 \%$ de comparecimentos.

Tendo em vista identificar o nível sócio-económico das gestantes atendidas, utilizou-se o critério adotado no hospital, no qual por uma pessoa que tenha de renda mensal até $\mathrm{Cr} \$ 522,00$ ficará na classe "CD"; de Cr\$ 523,00 a Cr\$ 870,00 ficará na classe "B"; de $\operatorname{Cr} \$ 871,00$ a Cr\$ 1.334,00 ficará na classe " $A$ "; de Cr\$1.335,00 e mais ficará na classe "A1".

Das 496 gestantes inscritas registrouse 101 da classe "A1"; 80 da classe "A", 106 da classe "B" e 209 da classe "CD". E importante salientar que a predominância foi da classe "CD", com 42,20\%, onde a renda familiar é de até $\operatorname{Cr} \$ 522,00$. Considera-se que a atenção de enfermagem, através de ações educativas a esse grupo de gestantes, é relevante.

Quanto à imunização anti-tetânica, aplicou-se 1.179 doses de Anatox Tetânico, sendo 425 primeiras doses e 328 imunizações.

Através da consulta de enfermagem, foram identificados problemas, sendo alguns tratados pela enfermeira, outros encaminhados ao médico, à maternidade ou a outros recursos da Comunidade. Embora o tratamento de enfermagem se caracterize pela atuação da enfermeira orientando, supervisionando, ajudando ou encaminhando a paciente, demonstrou-se no quadro abaixo os problemas identificados em que o tratamento foi basicamente através de ações educativas específicas e os encaminhados. Também, nos casos registrados como encaminhados, houve atuação da enfermeira orientando a conduta, local, horário, a que o profissional deveria se dirigir. (Quadro I). 
DUARTE, N. M. N., MUXFELDT, L. C. - O papel da enfermeira na assistência à gestante sadia, Rev. Bras. F.nf., R.J., 28 : $70-74,1975$.

\section{Q. U A D R O 1}

PROBLEMAS IDENTNFICADOS E TRATADOS NA CONSULTA DE ENFERMAGEM OBSTÉTRICA, de FEVEREIRO de 1973 a ABRIL DE 1975.

PROBLEMAS IDENTIFICADOS

Aumento excessivo de peso

Constipação intestinal
TRATOS P/ ENCAMIENFERMEIRA NHADOS
Erro alimentar

Dilatação precoce do colo uterino

Corrimento vaginal

Ansiedade

Edema discreto de M. M. S. S.

Dor abdominal

Verminose

Trabalho de Parto

Pré-eclampsia

Anemia Severa

Hipercinesia uterina precoce

Infecção urinária

Sedentarismo

Dentes em mau estado

Sangramento vaginal

Leucocitúria

Vômitos

Glicosúria

Diarréia

Pirose

Hemorróides

Desajuste sexual

Trabalho de parto prematuro

Escabiose

Tabagismo

Incompatibilidade $\mathrm{Rh}$

Perda excessiva de peso

Toxoplasmose

Hipoglicemia

Crises de agressividade

Gestante solteira

Tonturas

Alcoolismo $\therefore 15$

109
04

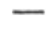


DUARTE, N.M.N. \& MUXFELDT, L.C. - O papel da Enfermeira na Assistência à gestante sadia.Rev. Bras. Enf.; 28 : 70-74, 1975.

\section{b) PERCEPÇÃO DAS GESTANTES QUANTO AO ATENDIMENTO DE ENFERMAGEM}

Através do preenchimento de questionários por 70 gestantes, que tiveram mais de 3 consultas de enfermagem, foi feita avaliação da receptividade quanto ao atendimento, tipo de orientação e comunicação da enfermeira, durante a consulta de enfermagem, bem como tipo de assistência que recebia antes da inscrição no HCPA. De 70 pacientes que opinaram, 34 receberam, anteriormente, assistência em consultório particular, 24 no INPS, 4 em consultório particular e INPS e 8 não receberam assistência alguma. Quanto a opinião sobre o atendimento realizado pela enfermeira obstétrica, 66 gestantes, ou seja, $94,28 \%$ registraram ser ótimo.

No que diz respeito à comunicação da enfermeira, durante a Consulta de Enfermagem, 67 gestantes registraram ser ótima, o que corresponde a 95,71\%. O restante, ou seja, $4,29 \%$, registraram como boa.

\section{c) RESULTADOS REFERENTES AO CURSO PSICOPROFILÁTICO}

Até o momento foram desenvolvidos 24 cursos, com uma média de 12 gestantes por curso. O total de gestantes inscritas foi de 300 , sendo $82,70 \%$ com re- gistro no HCPA e as demais, $17,30 \%$, não eram registradas no HCPA.

De 300 inscrições, 12 foram as desistências, sendo 5 ocasionadas por transferência de residência, 3 por parto prematuro e 4 por causa desconhecida.

O comparecimento às aulas foi de 2.563 presenças, ou seja, $89,76 \%$.

\section{IV - CONCLUSÃO}

No programa atualmente em execução no HCPA, a enfermeira assume grande responsabilidade, participando diretamente de atividades de promoção da saúde e proteção específica. Assume, também, um importante papel na avaliação das necessidades físicas, sócioeconômicas, emocionais e educativas das gestantes, através da Consulta de Enfermagem.

Ao desenvolver o programa de educação contínua, atividade esta de responsabilidade da enfermeira, ela estará dispensando à gestante oportunidade de maiores esclarecimentos e de contato mais direto com os profissionais.

Segundo a avaliação, concluiu-se que a participação da enfermeira em atenção direta à gestante sadia, dinamiza 0 Programa de Saúde Materna e dá oportunidade à gestante de alto risco ser atendida mais vezes pelo médico. Através de um maior equacionamento da atenção de enfermagem, pode-se aumentar a cobertura de programas dessa natureza na Comunidade.

\section{BIBLIOGRAFIA}

CHAVES, Mário. Saúde e Sistemas. Rio de Janeiro, R. J. Fundação Getúlio Vargas, 1972.

DU, GAS, Beverly W. - Tratado de Enfermería Práctica. - 2. ${ }^{\text {a }}$ ed. México, Interamericana, pg, 437, 1974.

HORTA, Wanda de Aguiar. Processo de Enfermagem.

São Paùo, 1972. Polígrafos.
MUXFELDT, Léa Cecília et al. O papel da Enfermeira na Assistência à Criança Sadia. - Programa Materno Infantil do Hospital de Clínicas de Porto Alegre. Porto Alegre, 1972. Polígrafo.

Manual de Normas e Rotinas do Serviço de Ginecologia e Obstetrícia, do Hospital de Clínicas de Porto Alegre - Porto Alegre, 1972. 